\title{
Alterations of Ocular Hemodynamics Impair Ophthalmic Vascular and Neuroretinal Function
}

\author{
Shu-Huai Tsai, Wankun Xie, Min Zhao, Robert H. Rosa, Jr, Travis W. Hein, and Lih Kuo
}

From the Department of Medical Physiology, College of Medicine, Texas A\&M University Health Science Center, Temple; and the Ophthalmic Vascular Research Program, Department of Ophthalmology, Scott \& White Eye Institute, Baylor Scott \& White Health, Temple, Texas

Accepted for publication

November 21, 2017.

Address correspondence to Lih Kuo, Ph.D., Department of Medical Physiology, Texas A\&M University Health Science Center, 702 SW H.K. Dodgen Loop, Temple, TX 76504. E-mail: 1kuo@ tamhsc.edu.

\begin{abstract}
Hypertension is associated with numerous diseases, but its direct impact on the ocular circulation and neuroretinal function remains unclear. Herein, mouse eyes were challenged with different levels of hemodynamic insult via transverse aortic coarctation, which increased blood pressure and flow velocity by $50 \%$ and $40 \%$, respectively, in the right common carotid artery, and reduced those parameters by $30 \%$ and $40 \%$, respectively, in the left common carotid artery. Blood velocity in the right central retinal artery gradually increased up to $40 \%$ at 4 weeks of transverse aortic coarctation, and the velocity in the left central retinal artery gradually decreased by $20 \%$. The fundus and retinal architecture were unaltered by hemodynamic changes. Endothelium-dependent vasodilations to acetylcholine and adenosine were reduced only in right (hypertensive) ophthalmic arteries. Increased cellularity in the nerve fiber/ganglion cell layers, enhanced glial fibrillary acidic protein expression, and elevated superoxide level were found only in hypertensive retinas. The electroretinogram showed decreased scotopic b-waves in the hypertensive eyes and decreased scotopic oscillatory potentials in both hypertensive and hypotensive eyes. In conclusion, hypertension sustained for 4 weeks causes ophthalmic vascular dysfunction, retinal glial cell activation, oxidative stress, and neuroretinal impairment. Although ophthalmic vasoregulation is insensitive to hypotensive insult, the ocular hypoperfusion causes neuroretinal dysfunction. (Am J Pathol 2018, 188: 818-827; https://doi.org/10.1016/j.ajpath.2017.11.015)
\end{abstract}

Retinal blood flow is regulated to match the high metabolic demand of the neural tissue and to maintain normal retinal function. ${ }^{1}$ Under physiological conditions, the ocular blood flow is maintained relatively constant despite changes in perfusion pressure, ${ }^{2-4}$ and hyperemia occurs in response to metabolic activation. ${ }^{5}$ This blood flow regulation phenomenon is achieved by altering ocular flow resistance through adjusting vascular diameter, in part, by the endotheliumdependent vasodilation mechanisms. ${ }^{6}$ For example, the increased release of local metabolites, such as adenosine $e^{7,8}$ and lactate, ${ }^{9,10}$ on metabolic activation can trigger vasodilation via activation of endothelial nitric oxide (NO) synthase (NOS). ${ }^{11-14}$ The washout of these vasodilators by increased blood flow, in association with increased perfusion pressure, can increase vascular resistance by triggering vasoconstriction, and vice versa. Thus, this vasomotor regulation mechanism plays an important role in keeping tissue blood flow constant in the face of transient fluctuations in systemic blood pressure.
Chronic elevation of systemic blood pressure is associated with many cardiovascular diseases. Population-based cohort studies have shown an increased incidence of arteriolar narrowing, arteriovenous nicking, hemorrhages, and microaneurysms in the retina of patients with hypertension. ${ }^{15,16}$ A positive correlation on prevalence of retinopathy and the severity of hypertension has long been recognized. ${ }^{17}$ In addition, oscillatory potential reduction in the electroretinogram (ERG) has been noted in hypertensive patients with mild or no funduscopic changes in the retina. ${ }^{18}$ Although these correlative clinical observations suggest a close relationship between hypertension and retinopathy, the

\footnotetext{
Supported by the Liles Macular Degeneration Research Fund (R.H.R.), Kruse Chair Endowment (L.K.), Baylor Scott \& White-Central Texas Foundation (L.K.), Ophthalmic Vascular Research Program of Baylor Scott \& White Health (L.K.), Retina Research Foundation (L.K.), and NIH grants R01EY018420 and R01EY024624 (T.W.H.).

Disclosures: None declared.
} 
direct impact of systemic blood pressure changes on ocular blood flow regulation and ophthalmic vasomotor function remains unknown. Moreover, temporal changes in retinal structure and neuronal function in response to altered ocular blood pressure and flow are incompletely understood.

To address the aforementioned issues, we performed transverse aortic coarctation (TAC) in mice by constricting the aorta between the brachiocephalic artery and the left common carotid artery (CCA). TAC generates different distributions of blood pressure and flow between the right and left carotid arteries, ${ }^{19-21}$ which feed into the central retinal artery (CRA) of the right and left eye via their respective ophthalmic arteries. With this TAC model, in the present study, we investigated the temporal blood flow regulation in the central retinal arteries and the endotheliumdependent vasomotor reactivity of ophthalmic arteries in response to the change in ocular perfusion pressure (hypertension in the right eye versus hypotension in the left eye) in the same animal. The impact of hemodynamic changes on retinal morphology and neural function was also examined.

\section{Materials and Methods}

\section{Animal Model and TAC}

The animal procedures were approved by the Institutional Animal Care and Use Committee, Baylor Scott \& White Health (Temple, TX), and conform to the Guide for the Care and Use of Laboratory Animals. ${ }^{22}$ All chemicals described later were purchased from Sigma-Aldrich (St. Louis, MO) unless otherwise noted. Male C57BL/6 mice, 6 to 8 weeks old, were anesthetized by i.p. injection of $100 \mathrm{mg} / \mathrm{kg}$ ketamine (Hospira, San Jose, CA) and $8 \mathrm{mg} / \mathrm{kg}$ xylazine (LLOYD Laboratories, Walnut, CA). A 2- to 3-mm left-sided thoracotomy was generated at the second intercostal space without cutting the ribs. ${ }^{20}$ Aorta between the brachiocephalic artery and left common carotid artery was located and looped with a 7.0 Prolene suture (Ethicon Inc., Somerville, NJ). With an overlaying 27-gauge needle, the aorta was then ligated. After removing the needle, the procedure produced a discrete region of stenosis in the aorta. The chest was then closed, and the mice were allowed to recover. A group of age-matched sham control mice received the same surgical procedures without ligating the aorta. The success of the aortic banding was confirmed by the development of left ventricular hypertrophy in 4 weeks, as shown in our previous study. ${ }^{23}$ This model generates hypertension in the right CCA and hypotension in the left CCA,${ }^{19-21}$ which feed into the right and left ophthalmic arteries and their downstream CRAs, respectively.

\section{Carotid Blood Pressure Measurement}

Mice were anesthetized with $100 \mathrm{mg} / \mathrm{kg}$ ketamine and $8 \mathrm{mg}$ / $\mathrm{kg}$ xylazine. A tracheostomy was performed in the midline of the neck. A small incision was made on the trachea, then a PE50 tube (20 mm long; Becton Dickinson and Company, Sparks, MD) was inserted and tied with the trachea to secure the airway. The CCA was isolated and cannulated with a PE10 tube connected to a pressure transducer (ADInstruments, Colorado Springs, CO), and the blood pressure was recorded using the PowerLab system (ADInstruments).

\section{Blood Flow Velocity Measurement}

Mice were anesthetized with 2.5\% (v/v) isoflurane (Piramal Healthcare, Mumbai, India) in $100 \%$ oxygen in a closed chamber, and the anesthesia was maintained with $2 \%$ isoflurane using a nose cone. Mice were taped on a procedure board maintained at $37^{\circ} \mathrm{C}$. The VisualSonics Vevo 2100 system (FujiFilm VisualSonics, Toronto, ON, Canada) and an MS-550D transducer with a frequency of $32 \mathrm{MHz}$ were used for blood velocity measurement. The CRA and CCA were located by color Doppler, and then the velocity waveform was recorded. Mean blood velocity was calculated by the Vevo LAB software version 1.7.1 (FujiFilm VisualSonics).

\section{Functional Assessment of Isolated Ophthalmic Arteries}

Mice were anesthetized with $100 \mathrm{mg} / \mathrm{kg}$ ketamine and $8 \mathrm{mg} /$ $\mathrm{kg}$ xylazine and then euthanized with heart removal. The eyes were removed with the retrobulbar tissue and placed in physiological salt solution $(\mathrm{NaCl}, 145.0 \mathrm{mmol} / \mathrm{L} ; \mathrm{KCl}, 4.7$ $\mathrm{mmol} / \mathrm{L} ; \mathrm{CaCl}_{2}, 2.0 \mathrm{mmol} / \mathrm{L} ; \mathrm{Mg}_{2} \mathrm{SO}_{4}, 1.17 \mathrm{mmol} / \mathrm{L}$; $\mathrm{NaH}_{2} \mathrm{PO}_{4}, 1.2 \mathrm{mmol} / \mathrm{L}$; glucose, $5.0 \mathrm{mmol} / \mathrm{L}$; pyruvate, 2.0 $\mathrm{mmol} / \mathrm{L}$; EDTA, $0.02 \mathrm{mmol} / \mathrm{L}$; and 3-morpholinopropane1 -sulfonic acid, $3.0 \mathrm{mmol} / \mathrm{L}$ ) with $1 \%$ albumin at $8^{\circ} \mathrm{C}$. The ophthalmic artery was isolated and then transferred to a tissue chamber and cannulated onto a pair of glass micropipettes and pressurized to $75 \mathrm{~cm} \mathrm{H}_{2} \mathrm{O}$ lumenal pressure without flow by two independent reservoir systems, as described previously. ${ }^{11,24}$ After developing stable basal tone at $36^{\circ} \mathrm{C}$ to $37^{\circ} \mathrm{C}$, vasodilations in response to the endothelium-dependent NO-mediated vasodilators acetylcholine (ACh; $10^{-8}$ to $\left.10^{-3} \mathrm{~mol} / \mathrm{L}\right)^{25}$ and adenosine $\left(10^{-8}\right.$ to $\left.10^{-5} \mathrm{~mol} / \mathrm{L}\right)^{11}$ and the endothelium-independent NOdonor sodium nitroprusside $\left(10^{-8} \text { to } 10^{-5} \mathrm{~mol} / \mathrm{L}\right)^{11}$ were examined. In another series of experiments, the vessels were incubated with the NOS inhibitor $N^{G}$-nitro-L-arginine methyl ester (L-NAME; $10^{-5} \mathrm{~mol} / \mathrm{L}$ ) for 30 minutes to assess the role of endothelial $\mathrm{NO}$ in the dilation of ophthalmic arteries. At the end of each experiment, the maximum diameter of the vessels was obtained at $10^{-4}$ $\mathrm{mol} / \mathrm{L}$ sodium nitroprusside in the presence of calcium-free physiological salt solution with $1 \mathrm{mmol} / \mathrm{L}$ EDTA.

\section{Fundus and Spectral Domain-0ptical Coherence Tomography Imaging}

Mice were anesthetized with isoflurane inhalation, and pupil dilation was achieved with $1 \%$ tropicamide eye drops 
(Bausch \& Lomb Inc., Rochester, NY) before image acquisition. The Spectralis HRA + OCT system with a 30-degree lens (Heidelberg Engineering, Heidelberg, Germany) was used to acquire fundus and cross-sectional images of the retina in vivo. A plano contact lens (Cantor + Nissel, Brackley, UK) was used for fundus imaging. The blue reflectance fundus image was taken under the excitation of a blue solid-state laser (wavelength, $488 \mathrm{~nm}$ ). Spectral domain-optical coherence tomography images were taken with a superluminescent diode at $870 \mathrm{~nm}$ as a low coherence light source. Scans were acquired at a speed of 40,000 A-scans per second, and each twodimensional B-scan contained up to 1536 A-scans. Images were analyzed with the Heidelberg Eye Explorer (HEYEX) software version 5.7 (Heidelberg Engineering). Diameters of retinal arterioles and venules in the fundus images were measured at a distance of one optic disc diameter from the optic disc margin.

\section{Histologic Examination}

Mice were anesthetized with $100 \mathrm{mg} / \mathrm{kg}$ ketamine and 8 $\mathrm{mg} / \mathrm{kg}$ xylazine and then euthanized with heart removal. Eyes were enucleated and fixed with $4 \%$ paraformaldehyde, dehydrated, and embedded into paraffin for conventional histology. Sections were deparaffinized, stained with hematoxylin and eosin, and examined with light microscopy. For immunohistochemistry and superoxide detection, the anterior segment and lens were removed and the posterior eyecup was embedded in Tissue-Tek O.C.T. Compound (Sakura Finetek, Torrance, CA) and cut into sections (10 $\mu \mathrm{m}$ thick) using a Leica CM1850 cryostat (Leica, Wetzlar, Germany). For glial cell staining, the sections were incubated with rabbit anti-glial fibrillary acidic protein primary antibody (1:500; Abcam, Cambridge, MA), followed by rhodamine-conjugated secondary antibody. Nuclei were counterstained with DAPI. For superoxide detection, sections were stained with 1 $\mu \mathrm{mol} / \mathrm{L}$ dihydroethidium for 10 minutes. Fluorescence images were taken using an IX83 inverted microscope (Olympus, Tokyo, Japan). Settings for image acquisition were identical in each set of experiments.

\section{Electroretinography}

Mice were dark adapted for at least 30 minutes and anesthetized with $100 \mathrm{mg} / \mathrm{kg}$ ketamine and $8 \mathrm{mg} / \mathrm{kg}$ xylazine. Pupils were dilated with $1 \%$ tropicamide eye drops. A contact electrode with $2.5 \%$ hypromellose lubricant gel (Goniovisc; HUB Pharmaceuticals, Rancho Cucamonga, CA) was placed on the cornea of each eye. Subcutaneously fixed needle electrodes served as references. Full-field ERGs were recorded with the ESPION System (Diagnosys LLC, Lowell, MA) with strobe flash intensities from 0.01 to 10 cd.second $/ \mathrm{m}^{2}$. An averaged response was recorded on the basis of five presentations.

\section{Statistical Analysis}

Vessel diameters were normalized to the percentage of maximal dilation. An oscillatory index was calculated from the ERG data by summing the first four peaks of the oscillatory potentials (OPs). Statistical analysis was performed using $t$-test or repeated-measures two-way analysis of variance, followed by Bonferroni multiple-range test when appropriate. $P<0.05$ was considered significant. The data are expressed as means \pm SEM.

\section{Results}

\section{Blood Flow Velocities in CCA and CRA}

In TAC mice, the blood pressure was increased by $50 \%$ and decreased by $30 \%$ in the right and left CCAs, respectively (Figure 1). The mean blood flow velocity in the right CCA increased by approximately $40 \%$ after 1 week of aortic banding and maintained at this level for 8 weeks. However, blood flow velocity in the right CRA remained unchanged after 1 week of banding and then gradually increased by $40 \%$ to a steady level at 4 weeks after operation (Figure 2A). Blood velocity in the left CCA decreased by $40 \%$ after 1 week of aortic banding and maintained for 8 weeks, but the corresponding velocity in the CRA gradually decreased by approximately $10 \%$ at 1 week after operation and continuously decreased to a steady level of $20 \%$ lower than control (ie, preoperation) after 4 weeks of banding (Figure 2B). There were no significant changes in both right and left CCA and CRA blood flow velocities in sham mice (Figure 2). Because ocular hemodynamics stabilized at 4 weeks after aortic banding, the assessments on ophthalmic vasomotor function, retinal morphology, and neural function were examined at this time point, as described later.

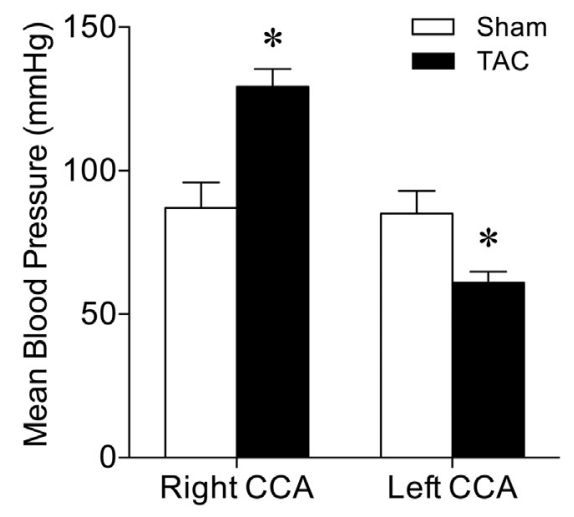

Figure 1 Carotid blood pressure in transverse aortic coarctation (TAC) mice. The blood pressure in the right common carotid artery (CCA) is elevated in the TAC mice compared with sham control. The blood pressure in the left CCA is decreased in the TAC mice. Data are expressed as means \pm SEM. $n=6$. ${ }^{*} P<0.05$ (t-test). 

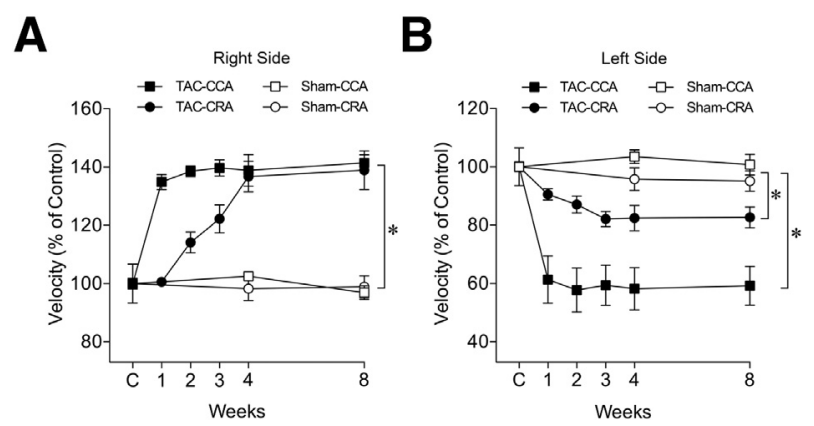

Figure 2 Carotid and central retinal artery blood flow velocities in transverse aortic coarctation (TAC) mice. A: The mean blood flow velocity in the right common carotid artery (CCA) is increased by $40 \%$ and maintained after 1 week after TAC (TAC-CCA). The blood flow velocity in the right central retinal artery (CRA) remains unchanged after 1 week and then slowly increases by $40 \%$ and reaches plateau at 4 weeks after operation (TACCRA). In sham mice, there are no significant changes in the right CCA (Sham-CCA) and CRA (Sham-CRA) blood flow. B: The mean blood velocity in the left CCA is decreased by $40 \%$ and maintained after 1 week after TAC (TAC-CCA). The blood velocity in the left CRA gradually decreases to a steady level of $20 \%$ lower than control at 4 weeks after TAC (TAC-CRA). In sham mice, there are no significant changes in left CCA (Sham-CCA) and CRA (Sham-CRA) blood flow. Data are expressed as means \pm SEM. $n=7$ (A and $\mathbf{B}$ ). ${ }^{*} P<0.05$ (repeated-measures two-way analysis of variance, with Bonferroni multiple-range test). $C$, control measurements before TAC or sham operation.

\section{Vasomotor Function of the Ophthalmic Artery}

Right and left ophthalmic arteries isolated from sham and TAC mice developed stable and comparable basal tone at 75 $\mathrm{cm} \mathrm{H}_{2} \mathrm{O}$ lumenal pressure, with no difference in their resting or maximum diameters (Table 1). The endotheliumdependent NO-mediated vasodilator $\mathrm{ACh}$ induced a concentration-dependent dilation of ophthalmic arteries isolated from either right (Figure 3A) or left (Figure 3B) eyes of sham mice, and this response was suppressed in the presence of the NOS inhibitor L-NAME (Figure 3, A and B). In the right ophthalmic arteries from mice with TAC for 4 weeks, the ACh-induced dilation was reduced and became insensitive to L-NAME (Figure 3A). The ACh-induced dilation of left ophthalmic arteries from TAC mice was comparable to that observed in sham mice (Figure 3B). Similarly, ophthalmic arterial dilations to another endothelium-dependent NO-mediated vasodilator adenosine were inhibited by L-NAME and were reduced in the right eye of TAC mice after 4 weeks of aortic banding (Figure 3C). The adenosine-induced dilation was not altered in the left ophthalmic arteries and remained sensitive to
L-NAME (Figure 3D). The dilations induced by the NO donor sodium nitroprusside were the same between right and left ophthalmic arteries from TAC mice, and their responsiveness was not different from sham mice (Figure 4).

\section{Fundus Appearance and Retinal Vascular Diameter}

No signs of fundus alterations, such as retinal hemorrhages or cotton wool spots, were found in either right or left eyes of mice subjected to TAC for 4 weeks (Figure 5A). Compared with sham mice, no marked changes in the diameters of retinal blood vessels, in terms of arteriovenous ratio, were found in either right or left eyes of these TAC mice (Figure 5B).

\section{Retinal Morphology and Histology}

The spectral domain-optical coherence tomography images showed no significant changes in the lamellar architecture of the retina in the TAC versus sham eyes (Figure 6A). Histologic examination disclosed increased cellularity in the nerve fiber layer/ganglion cell layer of the right eye of TAC mice but not of sham mice (Figure 6B). Immunofluorescence studies revealed increased glial fibrillary acidic protein expression in the nerve fiber layer, ganglion cell layer, inner plexiform layer, and outer plexiform layer of the right retina of TAC mice (Figure 6C). Moreover, dihydroethidium staining indicated an increased superoxide level in the three nuclear layers (ganglion cell layer, inner nuclear layer, and outer nuclear layer) of the right retina of TAC mice (Figure 6D).

\section{Retinal Electrophysiology}

Representative tracings of the full-field ERG under scotopic conditions are presented in Figure 7A. Compared with sham controls, there were no significant changes in the a-wave in both eyes of TAC mice (Figure 7B). Decreased b-wave amplitude was found only in the right eye of TAC mice (Figure 7C). The oscillatory index was significantly reduced in both right and left eyes of TAC mice (Figure 7D).

\section{Discussion}

The present study demonstrated the negative impact of hypertension and hypotension on retinal function before the

Table 1 Ophthalmic Arterial Diameter and Basal Tone

\begin{tabular}{|c|c|c|c|c|}
\hline \multirow[b]{2}{*}{ Variable } & \multicolumn{2}{|c|}{ Sham group $(n=8)$} & \multicolumn{2}{|c|}{ TAC group $(n=8)$} \\
\hline & Right eye & Left eye & Right eye & Left eye \\
\hline Resting diameter, $\mu \mathrm{m}$ & $80.1 \pm 2.9$ & $82.5 \pm 4.4$ & $86.3 \pm 2.7$ & $88.3 \pm 7.2$ \\
\hline Basal tone, \% & $75.2 \pm 1.7$ & $76.0 \pm 1.0$ & $76.7 \pm 1.2$ & $76.7 \pm 4.0$ \\
\hline
\end{tabular}

All data are expressed as means \pm SEM.

TAC, transverse aortic coarctation. 

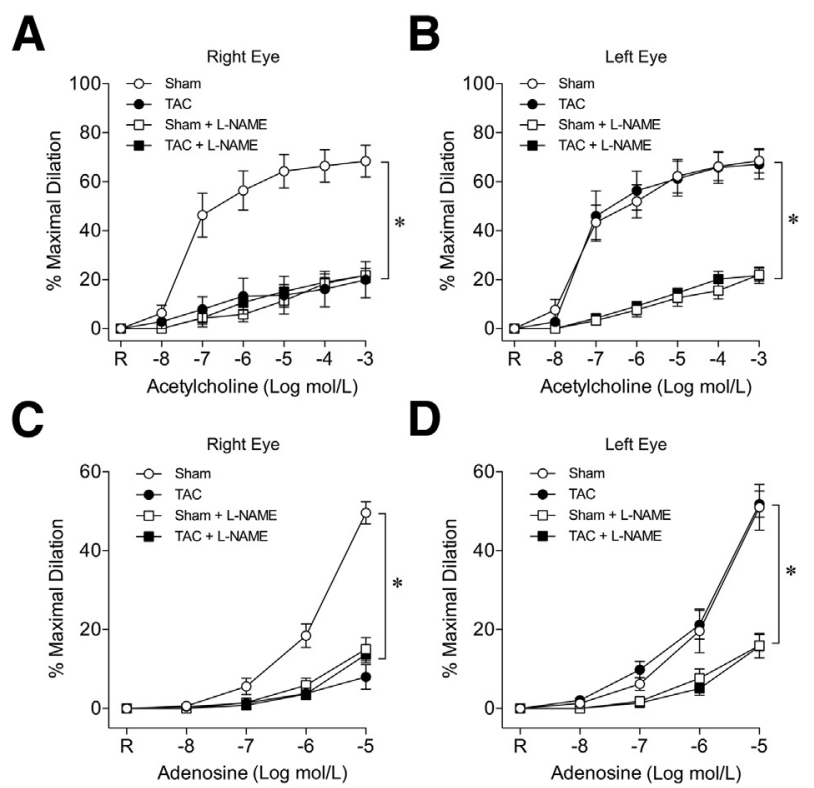

Figure 3 Deficiency of endothelium-dependent nitric oxide (NO) mediated ophthalmic arterial dilation in the hypertensive eye. A: Acetylcholine (ACh) induces concentration-dependent dilation of the ophthalmic artery from the right eye of sham mice (Sham). In the presence of N0 synthase inhibitor L-NAME $\left(10^{-5} \mathrm{~mol} / \mathrm{L}\right)$, the vasodilation is suppressed (Sham + L-NAME). Ophthalmic arterial dilation to ACh is reduced in the right (hypertensive) eye of mice after 4 weeks of transverse aortic coarctation (TAC), and L-NAME has no effect on this vasodilation (TAC + L-NAME). B: Ophthalmic arterial dilation to ACh remains unchanged in the left (hypotensive) eye of TAC mice after 4 weeks of banding (TAC). In the presence of L-NAME, the vasodilation is suppressed (TAC + L-NAME). C: Adenosine induces concentration-dependent dilation of the ophthalmic artery from the right eye of sham mice (Sham), and the vasodilation is suppressed by L-NAME (Sham + L-NAME). Adenosine-induced dilation is reduced in the right (hypertensive) eye of TAC mice after 4 weeks of banding (TAC) in a manner insensitive to L-NAME (TAC + L-NAME). D: In the left (hypotensive) eye of TAC mice, ophthalmic arterial dilation to adenosine remains unchanged (TAC) but is suppressed in the presence of L-NAME (TAC + L-NAME). Data are expressed as means \pm SEM. $n=7$ (A and C, TAC); $\mathrm{n}=6$ (all other groups). ${ }^{*} P<0.05$ (repeated-measures two-way analysis of variance, with Bonferroni multiple-range test). $R$, resting diameter.

onset of notable morphologic and structural changes in the retina. The chronic elevation of blood pressure in the ocular circulation leads to impairment of endothelium-dependent NO-mediated vasomotor regulation, microscopic alteration in the nerve fiber and ganglion cell layers, oxidative stress, and reduction in electrophysiological activity of the retina. Although the ophthalmic vasomotor function was not affected by hypotension, the retinal neural activity was compromised by chronic hypoperfusion. It appears that the ocular perfusion is not effectively regulated in response to chronic alterations in systemic blood pressure and, consequently, results in retinal dysfunction.

Although the incidence of retinopathy is reportedly increased in hypertensive patients in various clinical cohort studies, ${ }^{15,26-28}$ it remains unclear how this retinal abnormality occurs. Moreover, the temporal impacts of high blood pressure on ocular circulation and neuroretinal function are largely unknown. Recently, a hypertension rat model overexpressed with human renin and angiotensinogen genes showed the development of retinopathies with increased exudates, vascular leakage, and ganglion cell loss. ${ }^{29}$ However, the severe elevation of blood pressure (mean systolic pressure $>200 \mathrm{mmHg}$ ) in this rat model makes it difficult to incorporate the findings into current clinical observations. In the present study, the TAC model displayed differential distribution of blood pressure, as well as blood flow, between right and left CCAs feeding into the ocular circulation. This model allowed us to investigate the impact of both hypertension (right eye) and hypotension (left eye) on the ocular circulation in the same animal. The mean blood pressure in the right CCA was elevated approximately $50 \%$ from $87 \mathrm{mmHg}$ (sham control) to 129 $\mathrm{mmHg}$ (TAC). In contrast, the mean blood pressure in the left CCA was reduced by $30 \%$ from 85 to $60 \mathrm{mmHg}$ (Figure 1). The pressure difference between right and left CCAs in our TAC mice $(68 \mathrm{mmHg})$ was comparable to that reported by others (approximately $70 \mathrm{mmHg}$ ). ${ }^{20,30}$ The elevation of blood pressure in the right CCA was within the pathophysiological range commonly observed in hypertensive patients. The induction of hypertension by mechanical/ surgical intervention in the present study may minimize the confounding effects derived from genetic manipulation of the renin-angiotensin system, ${ }^{29}$ which has been shown to participate in the development of ocular pathophysiology independent of systemic blood pressure. ${ }^{31,32}$

In fact, the ocular perfusion is a function of pressure gradient between systemic blood pressure and the intraocular pressure (IOP). It has been documented in human subjects that the changes in IOP are positively correlated to the changes in systemic blood pressure. ${ }^{33-35}$ It is likely that the IOP in TAC mice was increased in the right (hypertensive) eye and decreased in the left (hypotensive) eye, which consequently reduced the impact of systemic blood pressure alteration on ocular perfusion. However, a

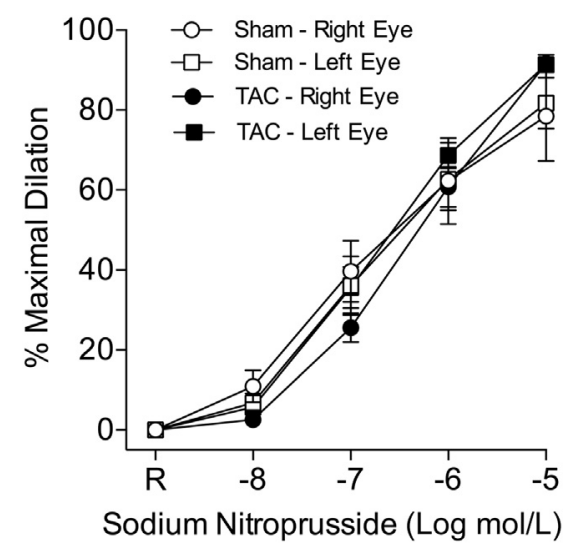

Figure 4 Endothelium-independent vasodilation. Ophthalmic arterial dilations to the nitric oxide donor sodium nitroprusside are not changed in either right or left eyes of transverse aortic coarctation (TAC) mice (repeated-measures two-way analysis of variance, with Bonferroni multiplerange test). Data are expressed as means \pm SEM. $n=6$. R, resting diameter. 
A

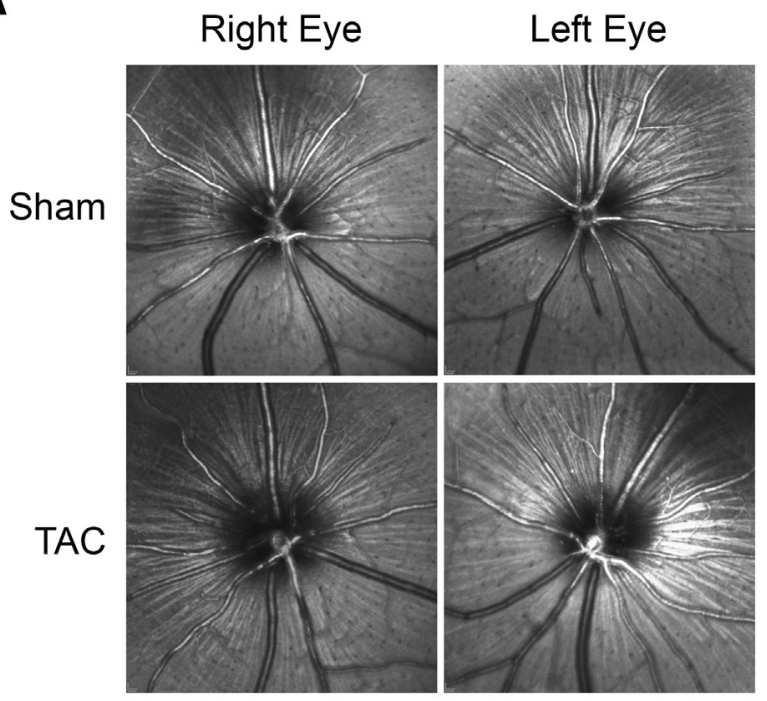

B

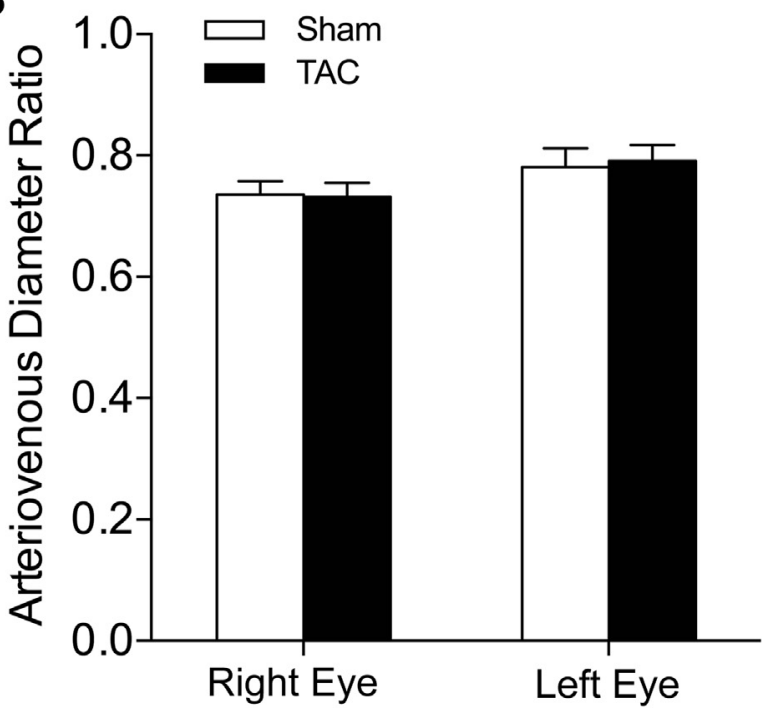

Figure 5 Fundus appearance and retinal vascular diameter in transverse aortic coarctation (TAC) mice. A: No signs of funduscopic changes are found in both right and left eyes at 4 weeks after TAC. B: The arteriovenous diameter ratio is not altered in the TAC mice compared with the sham control at 4 weeks after TAC ( $t$-test). Data are expressed as means \pm SEM (B). $n=6$ (B).

10-mmHg systemic blood pressure change can possibly lead to $0.2-$ to $0.4-\mathrm{mmHg}$ change in IOP. ${ }^{33-35}$ In our study, the elevation of right CCA blood pressure by $40 \mathrm{mmHg}$ in TAC animals might have caused 0.8 - to $1.6-\mathrm{mmHg}$ increase in IOP in the right eye. The decrease in IOP in the hypotensive eye of TAC mice might be $<1 \mathrm{mmHg}$ (in response to the $25-\mathrm{mmHg}$ decrease in the left CCA blood pressure). It appears that the impact of IOP changes on ocular perfusion was minimal in the present study.

The retinal blood flow is regulated to maintain constant perfusion during acute disturbance in blood pressure. , 3,36,37 $^{-3}$ However, to the best of our knowledge, the impact of chronic alteration in systemic blood pressure on temporal changes in retinal blood flow has not been previously explored. In the present study, the temporal change in CRA blood flow velocity was assessed against the values obtained from its upstream CCA. After TAC for 1 week, the blood flow velocity in the right CCA increased by $40 \%$ (Figure 2). By contrast, the downstream blood flow velocity in the CRA remained unchanged, suggesting an effort of blood flow regulation in the retinal vasculature to maintain a constant perfusion in the early phase of hypertension. However, after 1 week, the CRA blood flow velocity gradually increased and then plateaued at $40 \%$ above control. The percentage increase in CRA blood flow velocity was equivalent to that observed in its upstream CCA at steady state, indicating the failure of retinal flow regulation at the later phase of sustained hypertension. In the hypotensive state, the blood velocity in the left CCA decreased by $40 \%$ within 1 week of aortic banding (Figure 2). However, the flow velocity in the left CRA was gradually decreased by $10 \%$ at the first week of hypotension and then stabilized to $20 \%$ lower than control at 4 weeks after TAC. Because the relative reduction of flow velocity in the left CRA was not as pronounced as that of its upstream CCA, the retinal vasculature appears to preserve some degree of flow regulation under hypotension. Overall, our studies indicate that retinal blood flow can be fairly regulated in the early stage (ie, days to 1 week) of blood pressure disturbances, but this flow regulation mechanism gradually fails with time. It appears that the retinal flow regulation is less susceptible to chronic hypotension than to hypertension.

Because the CRA blood flow stabilized at 4 weeks after TAC, the assessments on vasomotor activity, retinal morphology, and function were performed at this time point. First, we further characterized the impact of hypertension and hypotension on ocular blood flow regulation by examining vasomotor activity of ophthalmic arteries using an isolated vessel approach. Many human ${ }^{5,12,38-41}$ and animal $^{13,14,42-44}$ studies have supported the physiological role of endothelial NO, synthesized by NOS, in regulation of the ocular circulation, especially under conditions with metabolic activation. ${ }^{5,38}$ In the present study, the neurotransmitter $\mathrm{ACh}^{6,45}$ and the metabolic vasodilator adenosine $7,8,11,14$ were used to test the endothelium-dependent NO-mediated vasodilator function in ophthalmic vasculatures in vitro. We found that both ACh- and adenosineinduced vasodilations were blocked by L-NAME in sham experiments (Figure 3), suggesting the major contribution of $\mathrm{NO}$ to these vasomotor responses. Interestingly, in TAC mice, dilations of the right ophthalmic artery to the same NO-mediated agonists were reduced to the level similar to that produced by L-NAME in sham mice. Furthermore, these hypertensive ophthalmic arteries became insensitive to L-NAME (Figure 3, A and C) but responded normally to the NO donor sodium nitroprusside (Figure 4). These data suggest that the vasodilations mediated by endothelial $\mathrm{NO}$ are specifically compromised in the hypertensive eyes. These findings are consistent with the clinical suggestion of endothelial NOS dysfunction in the retina of hypertensive 
A

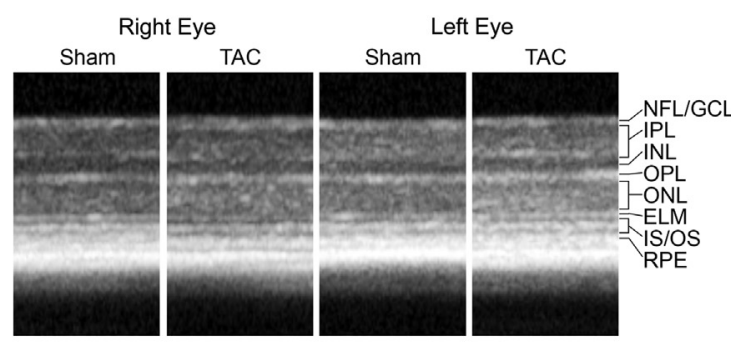

C

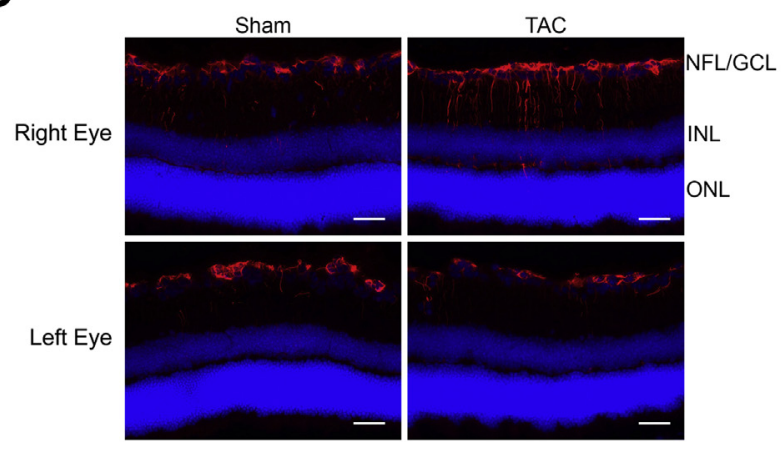

B

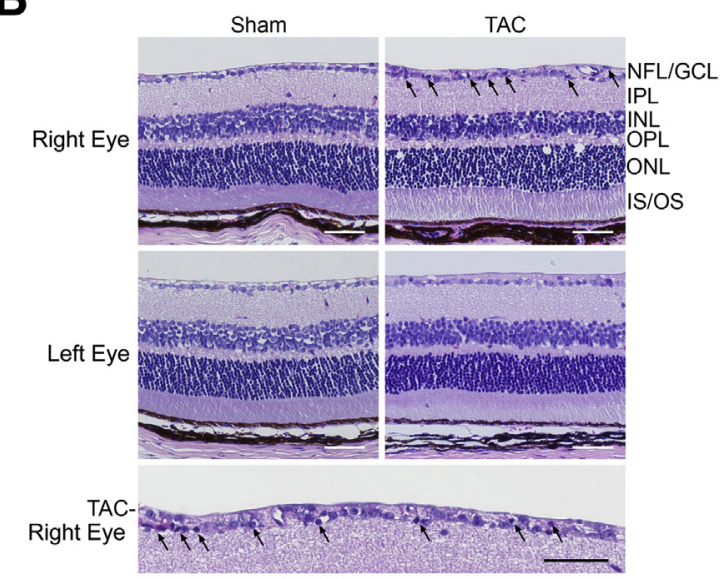

D

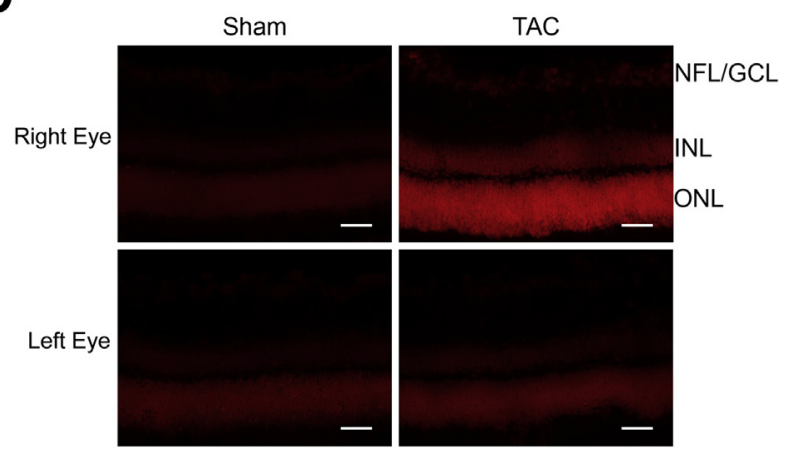

Figure 6 Cross-sectional structure of retina in transverse aortic coarctation (TAC) mice. A: Spectral domain-optical coherence tomography shows no significant changes in the lamellar architecture of the retina in the TAC versus sham eyes. B: Histologic examination reveals increased cellularity (arrows) in the nerve fiber layer (NFL) and ganglion cell layer (GCL) of the right eye (top panels) but not the left eye (middle panels) of TAC mice. The increased cellularity in the NFL and GCL of hypertensive eye is shown at higher magnification (bottom panel). C: Immunofluorescence signal of glial fibrillary acidic protein is enhanced in the NFL, GCL, and inner and outer plexiform layers (IPL and OPL, respectively) of the right retina of TAC mice. D: The superoxide level is increased in the right retina [GCL, inner nuclear layer (INL), and outer nuclear layer (ONL)] of TAC mice. No microscopic change is found in the left hypotensive eye of 4-week TAC mice. Scale bar $=50 \mu \mathrm{m}$ (B-D). ELM, external limiting membrane; IS/OS, photoreceptor inner/outer segment; RPE, retinal pigment epithelium.

patients. $^{39,40}$ Interestingly, enhanced oxidative stress and superoxide production in retinal tissue have been shown to be a hallmark of hypertension. ${ }^{46,47}$ It is speculated that the subsequent formation of hydrogen peroxide, in addition to the endothelial dysfunction, might contribute to the observed CRA blood flow dysregulation (ie, diminished vascular resistance and hyperperfusion) under chronic hypertension in our study, because this oxidant is a potent vasodilator in the microcirculation. ${ }^{48-50}$ By contrast, the ophthalmic artery from the hypotensive left eye showed comparable vasomotor function to that from the sham mice, suggesting that the endothelium-dependent NO-mediated vasodilation is preserved under sustained hypotension. Interestingly, the reduction of CRA blood flow velocity (ie, 20\%) was not as severe as that of the upstream CCA (ie, 40\%) (Figure 2), indicating that the intrinsic retinal blood flow regulation mechanism only partially restores the reduced perfusion in hypotension. It is likely that the development of some form of retinal microvascular dysfunction under chronic hypotension might account for the observed flow deficiency. Alternatively, the ability of flow regulation in the retina might be compromised when perfusion pressure is lowered beyond the critical range. This context is supported by the observed reduction in retinal arteriolar blood flow in a cat model of acute systemic hypotension by hemorrhage when blood pressure is reduced by $>30 \%,{ }^{14}$ similar to the extent seen in our current study. Further studies are needed to fully understand the pathophysiology of ocular hypotension.

Currently, the diagnosis of hypertensive retinopathy is primarily based on fundus appearance, and most of the observations are related to vascular abnormalities. ${ }^{28,51-54}$ In the present study, the vascular diameters in right (hypertensive) and left (hypotensive) eyes of the TAC mice were comparable to the sham mice, and there were no apparent fundus abnormalities (eg, arteriolar narrowing, exudates, hemorrhages, or microaneurysms) when the CRA blood flow velocity was stabilized at 4 weeks after aortic banding. Moreover, the arteriovenous diameter ratio appeared to be normal. However, our studies do not exclude the possible development of these pathophysiologies when the hemodynamic disturbance is prolonged beyond 4 to 8 weeks. Moreover, the cross-sectional structure of the retina examined by spectral domain-optical coherence tomography 
A

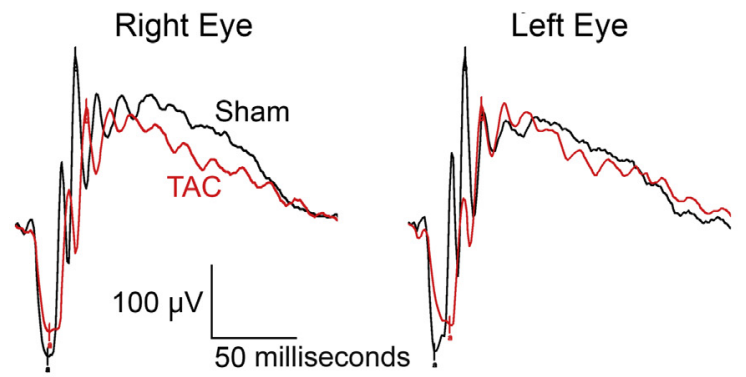

B
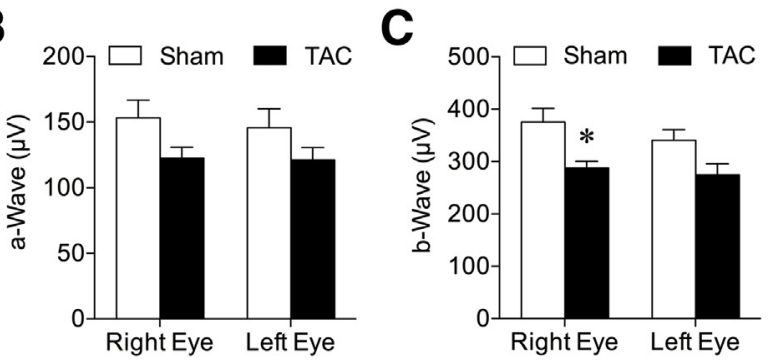

D

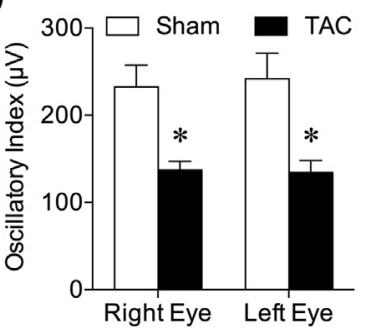

Figure 7 Electroretinography in transverse aortic coarctation (TAC) mice. A: Representative results of scotopic electroretinogram recorded from sham (black) and TAC (red) mice. B-D: Statistical analysis of the a-wave, b-wave, and oscillatory index was obtained from 6 sham and 10 TAC mice. B and C: The a-wave is not altered in TAC mice (B), but the b-wave amplitude is significantly reduced in the right eye of TAC mice (C). D: The oscillatory index is suppressed in both right and left eyes of TAC mice. Data are expressed as means \pm SEM (B-D). ${ }^{*} P<0.05$ ( $t$-test).

revealed no significant changes in the lamellar architecture of the retina in the TAC versus sham eyes (Figure 6A), despite the increased cellularity in the nerve fiber layer/ ganglion cell layer, as observed in histologic studies (Figure 6B). Interestingly, we found that retinal hypertension increased glial fibrillary acidic protein staining in Müller cell processes spanning from the internal limiting membrane to the outer plexiform layer of the hypertensive retina (Figure 6C), indicating the activation of Müller cells. ${ }^{55-57}$ It has been documented that macroglial cells (ie, astrocytes and Müller cells) are activated in response to neuroretinal damage for neuroprotection and repair. ${ }^{57,58}$ In conjunction with the observed increase in superoxide level in the hypertensive retina (Figure 6D), it is likely that the oxidative insult caused by chronic hypertension might lead to retinal dysfunction because of neuroretinal damage and macroglial activation.

Decreased ERG signals have been reported in hypertensive patients with mild or no funduscopic changes. ${ }^{18}$ In the present study, no significant change, albeit a trend of reduction, in the a-wave in both eyes of the mice after 4 weeks of aortic banding was observed. These data suggest that the function of photoreceptors remains intact under hemodynamic disturbances. On the other hand, a significant decrease in the scotopic b-wave amplitude occurred in the hypertensive eyes (Figure 7), indicating the impairment of inner retinal function, which might be associated with vascular dysfunction and/or macroglial activation (Figure 6, $\mathrm{B}$ and $\mathrm{C}$ ), as discussed in the preceding paragraphs. In line with current clinical observations, ${ }^{18,59-61}$ a significant decrease was observed in OPs in hypertensive eyes. Although the source of OPs is still unclear, it is considered to be generated from the middle retinal layers, ${ }^{62}$ possibly from amacrine and/or bipolar cells. ${ }^{63,64}$ It is likely that the flow dysregulation caused by chronic hypertension may subsequently lead to electrophysiological dysfunction in the inner/middle retinal layers related to glial cell activation and oxidative stress. This context is supported by the studies showing that acute hypertension/hyperperfusion can lead to elevated oxidative stress and brain injury ${ }^{65}$ and that antioxidant treatments may prevent the decrease in b-wave and OPs in the diabetic rat ${ }^{66}$ and also improve OPs in diabetic patients. ${ }^{67}$ Similar to hypertension, impaired retinal electrical function has been found in human diseases ${ }^{59,61,68}$ and in animal studies ${ }^{69,70}$ associated with retinal ischemia. It is likely that the cells residing in the inner nuclear layer, which are nourished by the deep retinal capillary plexus ${ }^{1}$ and responsible for the OP generation, ${ }^{62-64}$ are more susceptible to the insults from hypoperfusion. It has been shown that vitreous oxygen tension was significantly reduced when the mean arterial blood pressure was decreased by $>30 \%,{ }^{14}$ a level comparable to that observed in our current study. The prolonged reduction in CRA blood flow in the present study may possibly lead to retinal ischemia and explain the observed reduction in OPs in the hypotensive eyes.

\section{Conclusion}

TAC induces a differential distribution of blood pressure and blood flow between right and left eyes. In the right eye, the sustained elevation of blood pressure appears to exhaust the ability of blood flow regulation, possibly because of the impaired endothelial NO function and diminished microvascular resistance. These vascular changes may subsequently lead to ocular hyperperfusion, oxidative stress, glial cell activation, and neuroretinal dysfunction, which precede the macroscopic structural changes in the retina. In the left eye, the vasomotor regulation mediated by endothelial NO is preserved under hypotension, but the insufficient flow regulation (ie, ischemia) appears to compromise the neural retina function. We have demonstrated that a sustained ( 1 to 2 months) disturbance of systemic hemodynamics, either hypertension or hypotension, may be harmful to retinal function, even in the absence of funduscopic changes that are used to define retinopathy in the clinical setting. Our 
findings provide new insights into the development of functional retinopathy directly related to alterations of systemic blood pressure.

\section{References}

1. Kur J, Newman EA, Chan-Ling T: Cellular and physiological mechanisms underlying blood flow regulation in the retina and choroid in health and disease. Prog Retin Eye Res 2012, 31: 377-406

2. Movaffaghy A, Chamot SR, Petrig BL, Riva CE: Blood flow in the human optic nerve head during isometric exercise. Exp Eye Res 1998 67:561-568

3. Dumskyj MJ, Eriksen JE, Dore CJ, Kohner EM: Autoregulation in the human retinal circulation: assessment using isometric exercise, laser Doppler velocimetry, and computer-assisted image analysis. Microvasc Res 1996, 51:378-392

4. Riva CE, Titze P, Hero M, Movaffaghy A, Petrig BL: Choroidal blood flow during isometric exercises. Invest Ophthalmol Vis Sci 1997, 38:2338-2343

5. Garhofer G, Zawinka C, Resch H, Huemer KH, Dorner GT, Schmetterer L: Diffuse luminance flicker increases blood flow in major retinal arteries and veins. Vision Res 2004, 44:833-838

6. Pournaras CJ, Rungger-Brandle E, Riva CE, Hardarson SH, Stefansson E: Regulation of retinal blood flow in health and disease. Prog Retin Eye Res 2008, 27:284-330

7. Braunagel SC, Xiao JG, Chiou GC: The potential role of adenosine in regulating blood flow in the eye. J Ocul Pharmacol 1988, 4:61-73

8. Gidday JM, Park TS: Adenosine-mediated autoregulation of retinal arteriolar tone in the piglet. Invest Ophthalmol Vis Sci 1993, 34: 2713-2719

9. Winkler BS: Glycolytic and oxidative metabolism in relation to retinal function. J Gen Physiol 1981, 77:667-692

10. Winkler BS, Starnes CA, Sauer MW, Firouzgan Z, Chen SC: Cultured retinal neuronal cells and Muller cells both show net production of lactate. Neurochem Int 2004, 45:311-320

11. Hein TW, Yuan Z, Rosa RH Jr, Kuo L: Requisite roles of $A_{2 A}$ receptors, nitric oxide, and $\mathrm{K}_{\mathrm{ATP}}$ channels in retinal arteriolar dilation in response to adenosine. Invest Ophthalmol Vis Sci 2005, 46: 2113-2119

12. Hein TW, Rosa RH Jr, Yuan Z, Roberts E, Kuo L: Divergent roles of nitric oxide and rho kinase in vasomotor regulation of human retinal arterioles. Invest Ophthalmol Vis Sci 2010, 51:1583-1590

13. Hein TW, Xu W, Kuo L: Dilation of retinal arterioles in response to lactate: role of nitric oxide, guanylyl cyclase, and ATP-sensitive potassium channels. Invest Ophthalmol Vis Sci 2006, 47:693-699

14. Tani $T$, Nagaoka $T$, Nakabayashi S, Yoshioka T, Yoshida A: Autoregulation of retinal blood flow in response to decreased ocular perfusion pressure in cats: comparison of the effects of increased intraocular pressure and systemic hypotension. Invest Ophthalmol Vis Sci 2014, 55:360-367

15. Klein R, Klein BE, Moss SE, Wang Q: Hypertension and retinopathy, arteriolar narrowing, and arteriovenous nicking in a population. Arch Ophthalmol 1994, 112:92-98

16. Klein R, Klein BE, Moss SE: The relation of systemic hypertension to changes in the retinal vasculature: the Beaver Dam Eye Study. Trans Am Ophthalmol Soc 1997, 95:329-348; discussion 348-350

17. Yu T, Mitchell P, Berry G, Li W, Wang JJ: Retinopathy in older persons without diabetes and its relationship to hypertension. Arch Ophthalmol 1998, 116:83-89

18. Bellini G, Bocin E, Cosenzi A, Sacerdote A, Molino R, Solimano N, Ravalico G: Oscillatory potentials of the electroretinogram in hypertensive patients. Hypertension 1995, 25:839-841

19. Rockman HA, Ross RS, Harris AN, Knowlton KU, Steinhelper ME, Field LJ, Ross J Jr, Chien KR: Segregation of atrial-specific and inducible expression of an atrial natriuretic factor transgene in an in vivo murine model of cardiac hypertrophy. Proc Natl Acad Sci U S A $1991,88: 8277-8281$

20. Hill JA, Karimi M, Kutschke W, Davisson RL, Zimmerman K, Wang Z, Kerber RE, Weiss RM: Cardiac hypertrophy is not a required compensatory response to short-term pressure overload. Circulation 2000, 101:2863-2869

21. Hartley CJ, Reddy AK, Madala S, Michael LH, Entman ML, Taffet GE: Doppler estimation of reduced coronary flow reserve in mice with pressure overload cardiac hypertrophy. Ultrasound Med Biol 2008, 34:892-901

22. Committee for the Update of the Guide for the Care and Use of Laboratory Animals; National Research Council: Guide for the Care and Use of Laboratory Animals: Eighth Edition. Washington, DC, National Academies Press, 2011

23. Tsai SH, Lu G, Xu X, Ren Y, Hein TW, Kuo L: Enhanced endothelin-1/Rho-kinase signalling and coronary microvascular dysfunction in hypertensive myocardial hypertrophy. Cardiovasc Res 2017, 113:1329-1337

24. Kuo L, Davis MJ, Chilian WM: Endothelium-dependent, flowinduced dilation of isolated coronary arterioles. Am J Physiol 1990, 259:H1063-H1070

25. Laspas P, Goloborodko E, Sniatecki JJ, Kordasz ML, Manicam C, Wojnowski L, Li H, Patzak A, Pfeiffer N, Gericke A: Role of nitric oxide synthase isoforms for ophthalmic artery reactivity in mice. Exp Eye Res 2014, 127:1-8

26. Erden S, Bicakci E: Hypertensive retinopathy: incidence, risk factors, and comorbidities. Clin Exp Hypertens 2012, 34:397-401

27. Kabedi NN, Mwanza JC, Lepira FB, Kayembe TK, Kayembe DL: Hypertensive retinopathy and its association with cardiovascular, renal and cerebrovascular morbidity in Congolese patients. Cardiovasc J Afr 2014, 25:228-232

28. Katsi V, Marketou M, Vlachopoulos C, Tousoulis D, Souretis G, Papageorgiou N, Stefanadis C, Vardas P, Kallikazaros I: Impact of arterial hypertension on the eye. Curr Hypertens Rep 2012, 14: $581-590$

29. Reichhart N, Haase N, Crespo-Garcia S, Skosyrski S, Herrspiegel C, Kociok N, Fuchshofer R, Dillinger A, Poglitsch M, Muller DN, Joussen AM, Luft FC, Dechend R, Strauss O: Hypertensive retinopathy in a transgenic angiotensin-based model. Clin Sci (Lond) 2016, 130:1075-1088

30. Roman BB, Geenen DL, Leitges M, Buttrick PM: PKC $\beta$ is not necessary for cardiac hypertrophy. Am J Physiol Heart Circ Physiol 2001, 280:H2264-H2270

31. Wilkinson-Berka JL: Angiotensin and diabetic retinopathy. Int J Biochem Cell Biol 2006, 38:752-765

32. Fletcher EL, Phipps JA, Ward MM, Vessey KA, Wilkinson-Berka JL The renin-angiotensin system in retinal health and disease: its influence on neurons, glia and the vasculature. Prog Retin Eye Res 2010, 29:284-311

33. Klein BE, Klein R, Knudtson MD: Intraocular pressure and systemic blood pressure: longitudinal perspective: the Beaver Dam Eye Study. Br J Ophthalmol 2005, 89:284-287

34. Nakano T, Tatemichi M, Miura Y, Sugita M, Kitahara K: Long-term physiologic changes of intraocular pressure: a 10-year longitudina analysis in young and middle-aged Japanese men. Ophthalmology 2005, 112:609-616

35. Mitchell P, Lee AJ, Wang JJ, Rochtchina E: Intraocular pressure over the clinical range of blood pressure: Blue Mountains Eye Study findings. Am J Ophthalmol 2005, 140:131-132

36. Rassam SM, Patel V, Kohner EM: The effect of experimental hypertension on retinal vascular autoregulation in humans: a mechanism for the progression of diabetic retinopathy. Exp Physiol 1995, 80: $53-68$

37. Nagaoka T, Mori F, Yoshida A: Retinal artery response to acute systemic blood pressure increase during cold pressor test in humans. Invest Ophthalmol Vis Sci 2002, 43:1941-1945 
38. Dorner GT, Garhofer G, Kiss B, Polska E, Polak K, Riva CE, Schmetterer L: Nitric oxide regulates retinal vascular tone in humans. Am J Physiol Heart Circ Physiol 2003, 285:H631-H636

39. Delles C, Michelson G, Harazny J, Oehmer S, Hilgers KF, Schmieder RE: Impaired endothelial function of the retinal vasculature in hypertensive patients. Stroke 2004, 35:1289-1293

40. Michelson G, Warntges S, Harazny J, Oehmer S, Delles C, Schmieder RE: Effect of NOS inhibition on retinal arterial and capillary circulation in early arterial hypertension. Retina 2006, 26: 437-444

41. Nagaoka T, Takahashi A, Sato E, Izumi N, Hein TW, Kuo L, Yoshida A: Effect of systemic administration of simvastatin on retinal circulation. Arch Ophthalmol 2006, 124:665-670

42. Hein TW, Rosa RH Jr, Ren Y, Xu W, Kuo L: VEGF receptor2-linked PI3K/Calpain/SIRT1 activation mediates retinal arteriolar dilations to VEGF and shear stress. Invest Ophthalmol Vis Sci 2015, 56:5381-5389

43. Hein TW, Ren Y, Potts LB, Yuan Z, Kuo E, Rosa RH Jr, Kuo L: Acute retinal ischemia inhibits endothelium-dependent nitric oxidemediated dilation of retinal arterioles via enhanced superoxide production. Invest Ophthalmol Vis Sci 2012, 53:30-36

44. Yuan Z, Hein TW, Rosa RH Jr, Kuo L: Sildenafil (Viagra) evokes retinal arteriolar dilation: dual pathways via NOS activation and phosphodiesterase inhibition. Invest Ophthalmol Vis Sci 2008, 49: $720-725$

45. Haefliger IO, Flammer J, Luscher TF: Nitric oxide and endothelin-1 are important regulators of human ophthalmic artery. Invest Ophthalmol Vis Sci 1992, 33:2340-2343

46. Pinto CC, Silva KC, Biswas SK, Martins N, De Faria JB, De Faria JM: Arterial hypertension exacerbates oxidative stress in early diabetic retinopathy. Free Radic Res 2007, 41:1151-1158

47. Sicard P, Acar N, Gregoire S, Lauzier B, Bron AM, CreuzotGarcher C, Bretillon L, Vergely C, Rochette L: Influence of rosuvastatin on the $\mathrm{NAD}(\mathrm{P}) \mathrm{H}$ oxidase activity in the retina and electroretinographic response of spontaneously hypertensive rats. Br J Pharmacol 2007, 151:979-986

48. Thengchaisri N, Kuo L: Hydrogen peroxide induces endotheliumdependent and -independent coronary arteriolar dilation: role of cyclooxygenase and potassium channels. Am J Physiol Heart Circ Physiol 2003, 285:H2255-H2263

49. Yada T, Shimokawa H, Morikawa K, Takaki A, Shinozaki Y, Mori H, Goto M, Ogasawara Y, Kajiya F: Role of Cu,Zn-SOD in the synthesis of endogenous vasodilator hydrogen peroxide during reactive hyperemia in mouse mesenteric microcirculation in vivo. Am J Physiol Heart Circ Physiol 2008, 294:H441-H448

50. Yada T, Shimokawa H, Hiramatsu O, Shinozaki Y, Mori H, Goto M, Ogasawara Y, Kajiya F: Important role of endogenous hydrogen peroxide in pacing-induced metabolic coronary vasodilation in dogs in vivo. J Am Coll Cardiol 2007, 50:1272-1278

51. Keith NM, Wagener HP, Barker NW: Some different types of essential hypertension: their course and prognosis. Am J Med Sci 1974, 268:336-345

52. Wong TY, Mitchell P: Hypertensive retinopathy. N Engl J Med 2004, 351:2310-2317

53. Aissopou EK, Papathanassiou M, Nasothimiou EG, Konstantonis GD, Tentolouris N, Theodossiadis PG, Papaioannou TG, Sfikakis PP, Protogerou AD: The Keith-WagenerBarker and Mitchell-Wong grading systems for hypertensive retinopathy: association with target organ damage in individuals below 55 years. J Hypertens 2015, 33:2303-2309
54. Henderson AD, Biousse V, Newman NJ, Lamirel C, Wright DW, Bruce BB: Grade III or grade IV hypertensive retinopathy with severely elevated blood pressure. West J Emerg Med 2012, 13: $529-534$

55. Lieth E, Barber AJ, Xu B, Dice C, Ratz MJ, Tanase D, Strother JM; Penn State Retina Research Group: Glial reactivity and impaired glutamate metabolism in short-term experimental diabetic retinopathy. Diabetes 1998, 47:815-820

56. Luna G, Lewis GP, Banna CD, Skalli O, Fisher SK: Expression profiles of nestin and synemin in reactive astrocytes and Muller cells following retinal injury: a comparison with glial fibrillar acidic protein and vimentin. Mol Vis 2010, 16:2511-2523

57. de Hoz R, Rojas B, Ramirez AI, Salazar JJ, Gallego BI, Trivino A, Ramirez JM: Retinal macroglial responses in health and disease. Biomed Res Int 2016, 2016:2954721

58. Vecino E, Rodriguez FD, Ruzafa N, Pereiro X, Sharma SC: Glianeuron interactions in the mammalian retina. Prog Retin Eye Res 2016, 51:1-40

59. Bresnick GH, Korth K, Groo A, Palta M: Electroretinographic oscillatory potentials predict progression of diabetic retinopathy: preliminary report. Arch Ophthalmol 1984, 102:1307-1311

60. Ravalico G, Rinaldi G, Solimano N, Bellini G, Cosenzi A, Bocin E: Oscillatory potentials of the electroretinogram in hypertensive patients with different antihypertensive treatment. Doc Ophthalmol 1997, 94:321-326

61. Machalinska A, Kowalska-Budek A, Kawa MP, Kazimierczak A, Safranow K, Kirkiewicz M, Wilk G, Lubinski W, Gutowski P, Machalinski B: Association between asymptomatic unilateral internal carotid artery stenosis and electrophysiological function of the retina and optic nerve. J Ophthalmol 2017, 2017:4089262

62. McCulloch DL, Marmor MF, Brigell MG, Hamilton R, Holder GE, Tzekov R, Bach M: ISCEV Standard for full-field clinical electroretinography (2015 update). Doc Ophthalmol 2015, 130:1-12

63. Petit-Jacques J, Volgyi B, Rudy B, Bloomfield S: Spontaneous oscillatory activity of starburst amacrine cells in the mouse retina. $\mathrm{J}$ Neurophysiol 2005, 94:1770-1780

64. Phipps JA, Fletcher EL, Vingrys AJ: Paired-flash identification of rod and cone dysfunction in the diabetic rat. Invest Ophthalmol Vis Sci 2004, 45:4592-4600

65. Poulet R, Gentile MT, Vecchione C, Distaso M, Aretini A, Fratta L, Russo G, Echart C, Maffei A, De Simoni MG, Lembo G: Acute hypertension induces oxidative stress in brain tissues. J Cereb Blood Flow Metab 2006, 26:253-262

66. Feng Y, Wang R, Xu J, Sun J, Xu T, Gu Q, Wu X: Hydrogen-rich saline prevents early neurovascular dysfunction resulting from inhibition of oxidative stress in STZ-diabetic rats. Curr Eye Res 2013, 38: 396-404

67. Nebbioso M, Federici M, Rusciano D, Evangelista M, Pescosolido N: Oxidative stress in preretinopathic diabetes subjects and antioxidants. Diabetes Technol Ther 2012, 14:257-263

68. Tormene AP, Doro D, Mantovani E, Fabris S, Moro F: Electrophysiological findings in anterior ischemic optic neuropathy. Metab Pediatr Syst Ophthalmol (1985) 1989, 12:76-79

69. Jehle T, Wingert K, Dimitriu C, Meschede W, Lasseck J, Bach M, Lagreze WA: Quantification of ischemic damage in the rat retina: a comparative study using evoked potentials, electroretinography, and histology. Invest Ophthalmol Vis Sci 2008, 49:1056-1064

70. Luu CD, Foulds WS, Kaur C: Electrophysiological findings in a porcine model of selective retinal capillary closure. Invest Ophthalmol Vis Sci 2012, 53:2218-2225 